\title{
A novel individualized strategy for cryoballoon catheter ablation in patients with paroxysmal atrial fibrillation
}

\section{Jun Ding}

Tianjin Chest Hospital

Jing Xu

Tianjin Chest Hospital

\section{Wei Ma}

Tianjin Chest Hospital

\section{Bingwei Chen}

Tianjin Chest Hospital

\section{Peigen Yang}

Tianjin Chest Hospital

\section{Yu Qi}

Tianjin Medical University

Shan Sun ( $\nabla$ drss_sas@hotmail.com )

Tianjin Chest Hospital https://orcid.org/0000-0003-0763-0999

\section{Aijuan Cheng}

Tianjin Chest Hospital

\section{Research article}

Keywords: Atrial Fibrillation, Cryoablation, Catheter Ablation, Cryoballoon, Dosing

Posted Date: October 23rd, 2019

DOI: https://doi.org/10.21203/rs.2.16351/v1

License: (c) (1) This work is licensed under a Creative Commons Attribution 4.0 International License. Read Full License

Version of Record: A version of this preprint was published at BMC Cardiovascular Disorders on December 1st, 2019. See the published version at https://doi.org/10.1186/s12872-019-01295-1. 


\section{Abstract}

Background: The optimal dosage for cryoablation of atrial fibrillation (Cryo-AF) is still unknown. To evaluate the efficacy of an individualized freeze duration, we compared the clinical outcome of patients treated with a time-to-pulmonary vein isolation (TT-PVI) or temperature-guided ablation protocol to the outcome of patients treated with a conventional ablation protocol. Methods: A total of 164 consecutive patients were included in the study. One method employed was a conventional dosing protocol (at least 2 applications of 180 seconds each)(the Cryo-AF Conventional group $n=84$ ), and the second method had a shorter protocol that was based on the TT-PVI or achievement of $-40^{\circ} \mathrm{C}$ within 60 seconds (the Cryo-AF Dosing group $n=80$ ). Results: We treated 656 pulmonary veins (PVs) with 1420 cryotherapy applications. The mean number of applications per patient was $8.7 \pm 0.8$, with no difference between groups (Cryo-AF Conventional , $8.7 \pm 0.8$ versus Cryo-AF Dosing , $8.6 \pm 0.8 ; \mathrm{P}=0.359)$. The Cryo-AF Dosing group required significantly less total cryotherapy application time $(990.60 \pm 137.77$ versus $1501.58 \pm 89.60$ seconds; $P$ $<0.001)$ and left atrial dwell time $(69.91 \pm 6.91$ versus $86.48 \pm 7.03$ minutes; $P<0.001)$ than the Cryo-AF Conventional group. Additionally, the Cryo-AF Dosing group required significantly less total procedure time $(95.03 \pm 6.50$ versus $112.43 \pm 7.11$ minutes; $P<0.001)$. We observed acute ATP-induced or spontaneous vein electric reconnections in 13 veins (1.98\%) after 20 minutes. The reconnection rates between the Cryo-AF Conventional and Cryo-AF Dosing groups were similar in that $2.98 \%$ and $0.94 \%$ of the initially isolated veins were reconnected, respectively, $(P=0.061)$. There was no difference in the recurrence rate of free atrial arrhythmia after a 1-year follow-up, which were $78.75 \%$ in the Cryo-AF Dosing group versus $78.57 \%$ in the Cryo-AF Conventional group ( $P=0.978$ ). Conclusion: A novel Cryo-AF dosing protocol guided by temperature or the TT-PVI can be used to individualize an ablation strategy. This new protocol can lead to a significant reduction in duration of the procedure, the cryoenergy dosage and the left atrial dwell time. The procedure had equal safety and similar acute and 1-year follow-up outcomes compared to the conventional approach.

\section{Background}

Atrial fibrillation (AF) is a very universal sustained cardiac arrhythmia. Pulmonary vein (PV) isolation $(\mathrm{PVI})$ is a critical component of AF ablation. Cryoballoon (CB) ablation is highly effective in achieving PV isolation to treat $\mathrm{AF}^{1-3}$.

The second-generation $\mathrm{CB}$ (CB2, Arctic FrontAdvance ${ }^{\mathrm{TM}}$, Medtronic, Inc.) was released with technical developments that allowed a larger and more consistent zone of freezing on the balloon surface. These developments translated into critical improvements in clinical and procedural outcomes when compared to the previous generation ${ }^{4,5}$. However, as with all AF catheter ablation strategies, recurrence of arrhythmia and reconduction can arise throughout gaps when ablative lesions are incomplete, and conversely, complications can arise when collateral energy is transferred to accidental tissues. To prevent damage to extra cardiac structures, shorter times of application have been suggested. Currently used ablation strategies are often based on a fixed freeze-cycle duration of 180 seconds that is followed by a bonus freeze cycle of the same length after successful PVI. Currently, the ideal cryoablation of atrial 
fibrillation (Cryo-AF) dose is not well established, and predictors of durable electrical isolation are poorly known. Some clinical studies evaluated shorter freeze-cycle durations and determined whether the bonus application was actually necessary ${ }^{6-9}$. Prior research has suggested the time-to-pulmonary vein isolation (TT-PVI) procedure was a critical variable 7,9 .

This study evaluated the safety and efficacy of a shorter application strategy, based on the TT-PVI or

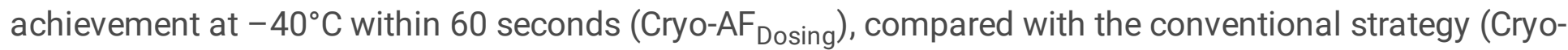
$\mathrm{AF}_{\text {Conventional). }}$.

\section{Methods}

\section{Patients}

This study was a single-centre, nonrandomized, open-label prospective clinical trial conducted at the Tianjin Chest Hospital. Patients with paroxysmal AF who received first-time CB ablation at the Tianjin Chest Hospital between October 2016 and January 2018 were enrolled in the trial. A total of 164 consecutive patients were included in the study. One method was a conventional dosing protocol. With this method, a 180-second freeze cycle was followed by a 180-second bonus freeze after acute PV isolation in the Cryo-AF $\mathrm{C}_{\text {Conventional }}$ group. The second method had a shorter protocol that was based on the TT-PVI or achievement of $-40^{\circ} \mathrm{C}$ within 60 seconds. Written informed consent was obtained from all patients. The inclusion criteria were age of 18 to 75 years, at least 2 paroxysmal AF episodes documented by electrocardiography, and no response to at least 1 antiarrhythmic drug. The exclusion criteria were acute coronary syndrome, persistent $A F$, left atrial size $>50 \mathrm{~mm}$, intracardiac thrombi, uncontrolled heart failure, and a history of a previous AF ablation procedure. Patients with a left common trunk or right middle PV were also excluded from the analysis. Three experienced operators performed the procedures. The same individual operators carried out both ablation strategies. The Institutional Review Board of Tianjin Chest Hospital approved the study protocol. The clinical baseline characteristics of the study patients are shown in Table 1.

\section{Ablation procedure}

Conscious sedation was established before the $\mathrm{CB}$ ablation procedure was performed. Vital parameters (oxygen saturation, blood pressure, pulse) were continuously monitored. During the ablation procedure, it was recommended that active clotting times be maintained between 300 and 350 seconds by intravenous administration of heparin. We used biplane fluoroscopy with $30^{\circ}$ right and $45^{\circ}$ left anterior oblique views. The electrophysiological study and cryoablation procedure were performed. A diagnostic catheter was delivered via the right internal jugular vein and positioned within the coronary sinus. An exchange wire was placed in the left superior PV after a single transseptal puncture access was made with an SL-1 sheath (St. Jude Medical, Inc.) using the modified Brockenbrough method (BRK-1, St. Jude Medical, Inc.). The PV anatomy and left atrial was visualized by using PV angiography. The SL-1 sheath 
was then replaced with a steerable sheath (FlexCath Advance ${ }^{\mathrm{TM}}$, Medtronic, Inc.). A 20-mm circular mapping catheter (Achieve ${ }^{\mathrm{TM}}$, Medtronic, Inc.) was used to guide the CB2 inside the left atrium (LA) and to try real-time recordings from the focused PV in all patients. Once a PV was inserted, the CB became inflated and approached to the vein ostium, and PV occlusion was confirmed by injection of contrast medium. Our standard set of lesions included the left superior pulmonary vein (LSPV), which was dealt with first, followed by the left inferior PV (LIPV), right inferior PV (RIPV), and right superior PV (RSPV). The Achieve catheter was placed and used during cryoablation; the TT-PVI was recorded "online" when the PV potentials have been dissociated from left atrial activity or completely disappeared. While ablating near the RSPV or RIPV, the phrenic nerve (PN) was stimulated with a bipolar catheter placed in the superior vena cava, and diaphragmatic stimulation was realized by pacing the ipsilateral phrenic nerve with a 1500-ms cycle and a 10-mA cycle. The operator periodically noticed the diaphragmatic contraction by using fluoroscopy and placed a hand on the abdomen of the patient to evaluate the strength of contraction. Cryoablation was terminated immediately if loss or reduction of the pacing capture was detected. The balloon was repositioned at the PV antrum if the PN function recovered and ablation was then reattempted. Radiofrequency (RF) ablation was used to complete PV isolation if PN function failed to recover within $10-15$ minutes.

In our study, we compared two cryotherapy application protocols. Cryoablation of 180 seconds was applied in the Cryo- $\mathrm{AF}_{\text {Conventional }}$ group until a bidirectional block of the vein was observed. Following successful PVI, an additional 180-second freeze cycle was applied to each PV. In the Cryo-AF ${ }_{\text {Dosing }}$ group, the freeze duration was modified relying the observed TT-PVI or balloon temperature. If a spontaneous TT-PVI was observed, cryoenergy applications lasted for the TT-PVI plus 60-90 seconds. If the TT-PVI was $<60$ seconds, a TT-PVI plus 60 -second freeze cycle was applied. If the TT-PVI was between 60 and 90 seconds, a TT-PVI plus 90 seconds freeze cycle was applied. We abandoned the cryoenergy applications that did not achieve TT-PVI within $90 \mathrm{~s}$. The CB was placed in an alternate position to achieve a suitable TT-PVI. Additionally, if the TT-PVI was unavailable, cryoenergy of 120-180 s was applied until the vein was blocked according to the time from the beginning of ablation to the balloon temperature achievement of $-40^{\circ} \mathrm{C}$. In other words, if the temperature reached $-40^{\circ} \mathrm{C}$ within 60 seconds, a 120 -second freeze cycle was applied, and if the temperature reached $-40^{\circ} \mathrm{C}$ within $60-90$ seconds, a 180 -second freeze cycle was carried out. A 120-second bonus operation was administered immediately in both cases, (Fig 1). If the balloon temperature fell beneath $-55^{\circ} \mathrm{C}$, the cryoapplication was terminated, and the balloon was placed in a different position at the PV antra to avoid ultra-cold temperatures. The endpoint of cryoablation for PVs was isolation. This was demonstrated by pacing the stimulation of the LA as well as the corresponding PV. The nadir cryotemperature and total cryotherapy time per vein during every cryoablation were recorded, as was the total procedure time. The main adverse events related to cryoablation, specifically, pericardial effusion or tamponade, transient ischaemic attack (TIA) or stroke, PN palsy, atrial-oesophageal fistulas, and symptomatic PV stenosis, were also recorded. Isolation of all PVs was reassessed by documentation of entrance- and exit-block after a 20-minute waiting period and in almost all cases following the administration of intravenous adenosine triphosphate (ATP). If PV reconnection was recorded, cryoenergy application of the reconnected PV was repeated. 


\section{Cryokinetics}

Three parameters of cryokinetics were introduced: TT-PVI, defined as the time from the beginning of the application to the moment the vein is blocked; time to $-40^{\circ} \mathrm{C}$, defined as theballoon freezing time from the beginning of the application to the moment of achievement of $-40^{\circ} \mathrm{C}$; and achievement $-40^{\circ} \mathrm{C}$ within 60 seconds, which was defined as the balloon freezing time; that is, from the beginning of the application to the achievement of $-40^{\circ} \mathrm{C}$ within 60 seconds.

\section{Postprocedural management and follow-up}

Patients were discharged from the hospital within 3 days of the procedure. Oral anticoagulation treatment was initiated on the evening of ablation and was continued for at least 2 months.

Antiarrhythmic drug (AAD) treatment was employed for 6 weeks. The patients were scheduled for followup visits at 1 and 3 months and then every 3 months after discharge from the hospital. The study considered a three-month blank period, and 12-lead electrocardiography and 24-hour Holter recordings were acquired at each follow-up visit. Any symptoms after ablation were deemed eligible for Holter monitoring. Furthermore, telephone calls were also executed throughout the follow-up. If atrial tachycardia (AT), AF, or atrial flutter (AFL) lasted more than 30 seconds, cryoablation was said to have failed, and the failure date was recorded as the date of recurrence. Otherwise, the treatment was regarded as successful.

\section{Statistical analysis}

Variables that composed a normal distribution were reported as the mean \pm standard deviation and variables were compared with a Student's t-test. The classification data were compared with the $\chi 2$ test or Fisher exact test. Kaplan-Meier method was used to predict the recurrence-free survival of AF, and the survival curve was compared with the log-rank test. The reported $P$ values were calculated using a twotailed test, and statistical significance was defined as $P<0.05$. All statistical analyses were performed using IBM SPSS statistical software (Version 19.0, SPSS).

\section{Results}

\section{Study population and procedural data}

Data from 164 consecutive patients who were treated with Cryo-AF for the first time were evaluated.

There were 80 patients who received Cryo-AF $\mathrm{F}_{\text {Dosing }}$ and 84 patients who received Cryo-AF $\mathrm{F}_{\text {Conventional. }}$ Table 1 shows the baseline data for the study population. There were no differences in the clinical characteristics between the two groups. All baseline characteristics were similar. 


\section{Acute procedural results}

We treated $656 \mathrm{PVs}$ with 1420 cryotherapy applications. During ablation, the potentials of PV in $83.23 \%$ of PVs could be monitored, with an average TT-PVI was $45.71 \pm 21.26$ seconds. In the Cryo-AF Dosing group, 320 PVs were treated. Of these 320 PVs, 269 (84.06\%) showed potentials during ablation and were treated with a cryotherapy dosage based on the TT-PVI. The other 51 (15.94\%) PV potentials were not visible. Specifically, of 51 PVs, 45 (14.06\%) PVs were treated with a cryotherapy dosage based on the achievement of $-40^{\circ} \mathrm{C}$ within 60 seconds, and $6(1.88 \%)$ PVs were treated with a cryotherapy dosage based on the achievement of $-40^{\circ} \mathrm{C}$ within $60-90$ seconds. Table 2 shows the procedure characteristics. Almost all (99.70\%) veins were successfully isolated. Only 1 left superior PV in the Cryo-AF $F_{\text {Dosing }}$ group and 1 right inferior $\mathrm{PV}$ in the $\mathrm{Cryo}_{\mathrm{A}} \mathrm{AF}_{\text {Conventional }}$ group remained reconnected after multiple attempts to isolation at the end of the procedure. The average number of applications per patient was $8.7 \pm 0.8$, there was no difference between the groups (Cryo- $\mathrm{AF}_{\text {Dosing, }}, 6.6 \pm 0.8$ versus Cryo-AF $\mathrm{F}_{\text {Conventional, }} 8.7 \pm 0.8 ; P=$ 0.359). The Cryo-AF $F_{\text {Dosing }}$ group required significantly less total cryotherapy application time $(990.60 \pm 137.77$ versus $1501.58 \pm 89.60$ seconds; $P<0.001)$ and left atrial dwell time $(69.91 \pm 6.91$ versus $86.48 \pm 7.03$ minutes; $P<0.001)$ than the Cryo-AF ${ }_{\text {Conventional }}$ group. Additionally, the Cryo- $\mathrm{AF}_{\text {Dosing }}$ group required significantly less total procedure time $(95.03 \pm 6.50$ versus $112.43 \pm 7.11$ minutes; $P<0.001)$ than the Cryo- $\mathrm{AF}_{\text {Conventional }}$ group. The average radiography exposure time was $11.57 \pm 0.88$ minutes and there was no difference between the two groups. The requirement for RF ablation did not differ between the two groups.

We observed acute ATP-induced or spontaneous vein electric reconnections in 13 veins (1.98\%) after 20

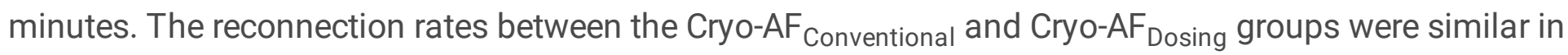
that $2.98 \%$ and $0.94 \%$ of the initially isolated veins were reconnected, respectively, $(P=0.061)$. After extra applications, all veins were successfully reisolated.

The results from the procedure (analysed per vein) are summarized in Table 3. The PV potentials monitored during the procedure were not different between the two groups. The average number of applications and the average TT-PVI were also not different between the two groups. The total cryotherapy times per vein in the Cryo- $\mathrm{AF}_{\text {Dosing }}$ group, however, were significantly shorter than in the Cryo$\mathrm{AF}_{\text {Conventional }}$ group (LSPV: $244.5 \pm 41.6 \mathrm{~s}$ versus $368.0 \pm 34.0 \mathrm{~s}, P<0.001$; LIPV: $244.0 \pm 45.5 \mathrm{~s}$ versus $375.0 \pm 45.0 \mathrm{~s}, P<0.001 ; \mathrm{RIPV}: 248.1 \pm 40.3 \mathrm{~s}$ versus $392.0 \pm 60.8 \mathrm{~s}, P<0.001$; RSPV: $236.8 \pm 41.9 \mathrm{~s}$ versus $366.7 \pm 21.2 \mathrm{~s}, P<0.001$, respectively). The average nadir temperatures in the right superior vein and left inferior vein were alike between the two groups. The nadir temperatures in the right inferior vein and left superior veins of the Cryo- $\mathrm{AF}_{\text {Dosing }}$ group, however, were lower than in the Cryo-AF $\mathrm{F}_{\text {Conventional }}$ group (LSPV: $-50.4^{\circ} \mathrm{C} \pm 3.7^{\circ} \mathrm{C}$ versus $-48.4^{\circ} \mathrm{C} \pm 5.6^{\circ} \mathrm{C}, P=0.007$; RIPV: $-49.7^{\circ} \mathrm{C} \pm 4.8^{\circ} \mathrm{C}$ versus $-46.8^{\circ} \mathrm{C} \pm 5.9^{\circ} \mathrm{C}, P=$ $0.001)$.

\section{Complications}


Complications, such as PN palsy, pericardial effusion, and groin vascular complications, were similar in two groups. In total, complications occurred in 4 of $80(5 \%)$ patients in the Cryo-AF $\mathrm{F}_{\text {Dosing }}$ group and 6 of $84(7.14 \%)$ patients in the Cryo- $\mathrm{AF}_{\text {Conventional }}$ group $(P=0.566)$. There were no incidences of stroke, atriooesophageal fistula, cardiac tamponade, or death during follow-up (Table 4).

\section{Follow-up}

The 164 patients assigned to intervention were monitored with a 24-hour Holter continuous electrocardiogram recording for each follow-up visit. There was no difference in the recurrence rate of free atrial arrhythmia between the two groups after three 3-month blanking period and after a 1-year followup. In addition, there were no significant differences between the $\mathrm{Cryo}_{\mathrm{A}} \mathrm{AF}_{\mathrm{Dosing}}$ and Cryo-AF $\mathrm{F}_{\text {Conventional }}$ groups in freedom from recurrent atrial arrhythmias at 3,6, 9, or 12 months (Table 5). Moreover, the Kaplan-Meier estimated freedom from atrial arrhythmias after the procedure did not differ significantly between the 2 groups (log-rank $P=0.956)$. Kaplan-Meier curves indicating freedom from atrial arrhythmias are shown in Fig 2.

\section{Discussion}

To the best of our knowledge, this was the first study that focused on a novel strategy for CB catheter ablation in patients with paroxysmal AF. This new strategy was based on a multiparametric evaluation (TT-PVI or achievement of $-40^{\circ} \mathrm{C}$ within $60 \mathrm{~s}$ ). In previous studies, according to the TT-PVI single parameter, $14.1-40 \%$ of the pulmonary vein potentials were unable to be recorded, and individualized cryoablation was not available $e^{7,9,10}$. In this study, with combined consideration of the balloon temperature and TT-PVI, nearly $100 \%$ of the pulmonary veins were treated with an individualized cryoablation strategy. The main aim of our study was to establish a simple and concise procedure, as well as an effective cryoablation strategy that could be easily implemented and consistantly replicated during Cryo-AF with different operators.

This study was a nonrandomized, single-center, open-label prospective analysis of the acute and longterm outcomes of cryotherapy used in patients with symptomatic AF. The strategy used a novel ablationdosing algorithm that was guided by the TT-PVI or temperature. The main findings of this study were: first, achievement of $-40^{\circ} \mathrm{C}$ within $60 \mathrm{~s}$ and TT-PVI are important considerations for both the efficacy and efficiency of cryoablation. This result indicated that TT-PVI can be used to individualize the ablation dosing protocol and reduce the freezing interval during Cryo-AF. Second, compared with previous investigations, this study increased the biophysics of ablation (achievement of $-40^{\circ} \mathrm{C}$ within 60 seconds) to further expand the application range of cryoablation dosing. PVs ablation in our study group was applied with a nearly $100 \%$ individualized cryotherapy-dosing protocol. Third, the temperature-guided dosing algorithm or TT-PVI increased the efficiency of cryotherapy, which allowed for a shorter cryotherapy time and reduced left atrial dwell and procedure times. However, the rates of freedom from 
recurrent AF during long-term follow-up and complications were the same between the two cryoablation strategies.

Various procedures and biophysical indicators that were previously reported, as well as predictors of acute and persistent PV isolation, were carefully evaluated during the development of the expected algorithm.Finally, based on available data, we developed the recommended dosing regimen, which depended on the TT-PVI or achievement of $-40^{\circ} \mathrm{C}$ within 60 seconds.

The TT-PVI has been identified as a prognostic indicator for PVI durability in CB ablation. In this previous study, the TT-PVI dosing strategy was used to determine the minimal effective time required to create a transmural lesion. A recent study conducted on animal models concluded that the CB2 achieved sustained PVI and that the duration of the freezing cycle was significantly shortened. In the canine model, effective PVI was found in the shortest administration group (TT-PVI +60 seconds), with an average total ablation time of approximately 90 seconds $^{11}$. Several studies have evaluated whether the TT-PVI dosing strategy could reduce cryoapplications and quantify ablation energy. The Individualized Cryoballoon Energy Trial (ICE-T) documented comparable outcomes when the freezing time was reduced. In the ICE-T, 50 subjects were treated with a standard freezing protocol of 240 seconds and an additional freezing protocol of 240 seconds. The group was compared to 50 patients who were treated with the TT-PVI dosing strategy. A bonus application was administered only if TT-PVI was $>75$ seconds. For $88 \%$ of PVs, a single-freeze protocol was used and TT-PVI was observed in $79 \%$ of PVs regardless of cohort designation. There were no differences in recurrence of AF during 1-year follow-up ${ }^{12}$. Aryana et al showed the biophysical and procedural characteristics of 112 patients who underwent a secondary procedure after CB2 cryotherapy of AF and PVI in 60 seconds were predictive of continued success ${ }^{13}$. Ciconte et al also suggested that TT-PVI be regarded as an independent predictor of atrial arrhythmia recurrences ${ }^{8}$. Ferrerode-Loma-Osorio et al performed a TT-PVI-guided dosing procedure in 69 patients compared with 68 control patients who underwent cryotherapy using a usual procedure. The authors found that the dosing procedure was associated with a shorter total ablation time ( $19.4 \pm 4.3$ versus $28.3 \pm 7$ minutes; $P<0.001)$, as well as left atrial dwell time ( $92 \pm 23$ versus $104 \pm 25$ minutes; $P<0.01)$ and total procedural time $(85 \pm 25$ versus $97 \pm 22$ minutes; $P<0.01)$, than that of the conventional procedure. The overall incidence of adverse events and 12 -month freedom from all atrial arrhythmia recurrence $(78.3 \%$ vs $79.4 \% ; P=0.869)$ were similar ${ }^{9}$.

When TT-PVI was not achievable, the balloon temperature could be used to guide ablation. Cryosurgical animal studies have demonstrated that maximal tissue damage occurs when a temperature below $40^{\circ} \mathrm{C}$ is obtained with a rapid temperature $\operatorname{drop}\left(>10^{\circ} \mathrm{C} / \mathrm{min}\right)^{14}$. Recent articles have reported that achieving $-40^{\circ} \mathrm{C}$ in 60 seconds is an independent predictor of durable $\mathrm{PVI}{ }^{15,16}$. lacopino et al compared 52 patients with temperature-assisted cryoablation to 52 patients with TT-PVI-guided cryoablation. The studies showed that a temperature-guided strategy that was based on achieving $-40^{\circ} \mathrm{C}$ within $60 \mathrm{~s}$ without real-time recordings was successful in producing PVI. Acute PVI was obtained in $99 \%$ of the PVs for the temperature-assisted cryoablation cohort. There was no difference in recurrence at a mean follow- 
up of 12 months between the two groups ( $85 \%$ vs. $88 \%$, respectively; $P=0.56)^{15}$. Giuseppe Cicon's research revealed that achievement of $-40^{\circ} \mathrm{C}$ within the first minute could independently predict durable $\mathrm{PVI}^{16}$. In a study by Wei et al., achievement of $-40^{\circ} \mathrm{C}$ within $60 \mathrm{~s}$ was observed in $67 \%$ of the PVs and

there was no association with acute reconnections, which was consistent with previous investigations ${ }^{17}$.

We designed a TT-PVI or temperature-based individualized protocol. In a conventional approach, some fixed cryoablation times may result in either insufficient or excessive durations. Prolonging the ablation over this duration did not result in further increases in the lesion depth or volume. Therefore, a longer than required ablation time may not only prolong the operation times but may also increase the risk of complications in important extracardiac structures, such as the esophagus, bronchial trees, or the PN. The use of a novel Cryo-AF individualized strategy may provide a more tailored approach that balances the effects of ablation while avoiding unnecessary over-ablation in thin-walled high-risk areas. There was a low incidence of severe events when this new procedure was used, and there was no difference between the two groups in the incidence of severe events. The TT-PVI or temperature-guided ablation protocol significantly reduced left atrial dwell, ablation, and procedure times. Our data could not deduce whether this finding translates into a reduction in adverse events, because overall adverse events were very low and the number of patients in this study was still too small to confidently estimate the incidence of complications. Further studies with a larger samples of patients are necessary in order to evaluate any potential reduction in complication rates. In contrast, insufficient freeze times may result in a higher recurrence rate of AF. In this study, a second segmental ablation was performed in PVs to ensure a wider range circumferential lesion to increase operational effectiveness. We therefore strongly suggest that the duration of cryoapplications should be guided through a systematic approach that is tailored based on objective and quantifiable measures of cryoablation during Cryo-AF. A TT-PVI or temperature-based individualized protocol has the potential to enhance PVI results and deserves further study. Future studies could determine whether patient-specific factors can be used to derive truly individualized optimal cryoablation dosages for each patient.

\section{Limitations}

The findings of this study are based on a single-centre experience and only recruit a limited number of patients in a non-random manner. We cannot rule out unknown confounding variables because the treatment assignment was nonrandomized. Randomized studies of a large number of patients may be required in the future to show the long-term effect of a TT-PVI or temperature-guided strategy.

\section{Conclusions}

A novel Cryo-AF dosing protocol guided by temperature or the TT-PVI can be used to individualize an ablation strategy. This new protocol can lead to a significant reduction in duration of the procedure, the cryoenergy dosage and the left atrial dwell time. The procedure had equal safety and similar acute and 1year follow-up outcomes compared to the conventional approach. 


\section{Declarations}

AF:atrial fibrillation; PV:pulmonary vein; PVI:pulmonary vein isolation;CB:cryoballoon; $\mathrm{CB} 2$ : secondgeneration CB; Cryo-AF:cryoablation of atrial fibrillation; RF:radiofrequency; TT-PVI:time-to-pulmonary vein isolation

\section{Ethics approval and consent to participate}

The study was approved by the Ethics Committee of Tianjin Chest Hospital before

the start of the work, and each patient signed a written consent form.

\section{Consent for publication}

Not applicable.

\section{Availability of data and materials}

The datasets generated and analysed during the current study are notpublicly available due to the Tianjin Chest Hospital regulations, but are availablefrom the corresponding author on reasonable request.

\section{Competing interests}

The authors declare that they have no competing interests.

\section{Funding}

This study was sponsored by the Science and Technology Fund of Tianjin Chest Hospital (2018-XKC09).

\section{Authors' contributions}

JD, JX and SS contributed to the conception or design of the work. JD,WM, BWC,PGY,YQ and AJC contributed to the acquisition, analysis, or interpretation of data. JD drafted the manuscript. JX and SS critically revised the manuscript. All authorsread and approved the final manuscript.

\section{Acknowledgements}


None.

\section{Tables}

Table 1 Baseline characteristics of the patients

\begin{tabular}{llll}
\hline Characteristic & Cryo-AF $_{\text {Dosing }}(\mathrm{n}=80)$ & Cryo-AF $_{\text {Conventional }}(\mathrm{n}=84)$ & $P$ \\
\hline Age (y) & $61 \pm 7$ & $59 \pm 11$ & 0.110 \\
Sex-maleGender & $45(56 \%)$ & $48(57 \%)$ & 0.908 \\
BMI (kg/m $\left.{ }^{2}\right)$ & $25.8 \pm 3.1$ & $26.1 \pm 3.5$ & 0.557 \\
Hypertension & $43(54 \%)$ & $42(50 \%)$ & 0.631 \\
Diabetes Mellitus & $12(15 \%)$ & $13(15 \%)$ & 0.932 \\
Stroke/TIA & $7(9 \%)$ & $13(15 \%)$ & 0.188 \\
CAD & $23(29 \%)$ & $20(24 \%)$ & 0.472 \\
CHA 2 DS 2 -VASc Score & $1.7 \pm 1.4$ & $1.7 \pm 1.4$ & 0.809 \\
LA Diameter (mm) & $38.0 \pm 3.4$ & $38.8 \pm 4.6$ & 0.222 \\
LVEF (\%) & $61.8 \pm 4.3$ & $60.8 \pm 4.5$ & 0.167 \\
\hline
\end{tabular}

Values are given as mean \pm SD or $\mathrm{n}(\%)$, unless otherwise indicated.There are no different characteristics between both groups. $\mathrm{BMI}=$ body mass index; $\mathrm{CAD}=$ coronary artery disease; $\mathrm{CHA}_{2} \mathrm{DS}_{2}-\mathrm{VASc}=\mathrm{score}$ for $\mathrm{AF}$ and stroke risk; LA=left atrial; LVEF=left ventricular ejection fraction; TIA=transient ischemic attack.

Table 2 Procedural Characteristics

\begin{tabular}{llll}
\hline & Cryo-AF $_{\text {Dosing }}(\mathrm{n}=80)$ & Cryo-AF $_{\text {Conventional }}(\mathrm{n}=84)$ & $P$ \\
\hline PV isolated, $\mathrm{n}(\%)^{\dagger}$ & $319(99.69 \%)$ & $335(99.70 \%)$ & 0.972 \\
${\text { RF ablation, } \mathrm{n}(\%)^{\dagger}}^{\dagger}$ & $1(0.31 \%)$ & $1(0.30 \%)$ & 0.972 \\
PV potentials, $\mathrm{n}(\%)^{\dagger}$ & $269(84.06 \%)$ & $277(82.44 \%)$ & 0.578 \\
PV acutely reconnected, $\mathrm{n}(\%)^{\dagger}$ & $3(0.94 \%)$ & $10(2.98 \%)$ & 0.061 \\
Total applications per patient, $\mathrm{n}$ & $8.6 \pm 0.8$ & $8.7 \pm 0.8$ & 0.359 \\
Total cryotherapy time per patient,s & $990.60 \pm 137.77$ & $1501.58 \pm 89.60$ & $<0.001$ \\
LA dwell time, min & $69.91 \pm 6.91$ & $86.48 \pm 7.03$ & $<0.001$ \\
Total procedure time, min & $95.03 \pm 6.50$ & $112.43 \pm 7.11$ & 0.000 \\
Radiography exposure time,min & $11.49 \pm 0.85$ & $11.65 \pm 0.91$ & 0.248 \\
\hline
\end{tabular}

Values are given as mean \pm SD or $\mathrm{n}(\%)$, unless otherwise indicated.

Cryo- $\mathrm{AF}=$ cryoablation of atrial fibrillation; $\mathrm{LA}=$ left atrial; $\mathrm{PV}=$ pulmonary vein; $\mathrm{RF}=$ radiofrequency. 


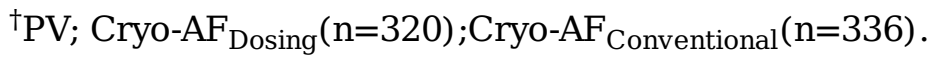

Table 3 Acute Results of Cryoablation Procedure

\begin{tabular}{|c|c|c|c|}
\hline Vein & Cryo- $\mathrm{AF}_{\text {Dosing }}(\mathrm{n}=80)$ & Cryo- $\mathrm{AF}_{\text {Conventional }}(\mathrm{n}=84)$ & $P$ \\
\hline \multicolumn{4}{|l|}{ Vein(LSPV) } \\
\hline PV potentials, n (\%) & $77(96.25 \%)$ & 79(94.05\%) & 0.513 \\
\hline TT-PVI, s & $49.0 \pm 20.7$ & $52.9 \pm 22.5$ & 0.263 \\
\hline Nadir temperature, ${ }^{\circ} \mathrm{C}$ & $-50.4 \pm 3.7$ & $-48.4 \pm 5.6$ & 0.007 \\
\hline Total cryotherapy time per vein, $s$ & $244.5 \pm 41.6$ & $368.0 \pm 34.0$ & $<0.001$ \\
\hline 2 lesions per vein, n (\%) & 73(91.25\%) & 73(86.90\%) & 0.374 \\
\hline Late/ATP reconnection,n (\%) & $1(1.25 \%)$ & $2(2.38 \%)$ & 0.585 \\
\hline \multicolumn{4}{|l|}{ Vein(LIPV) } \\
\hline PV potentials,n( \%) & $69(86.25 \%)$ & $70(82.35 \%)$ & 0.603 \\
\hline TT-PVI, s & $45.2 \pm 21.7$ & $44.7 \pm 23.2$ & 0.124 \\
\hline Nadir temperature, ${ }^{\circ} \mathrm{C}$ & $-45.5 \pm 3.8$ & $-44.2 \pm 4.5$ & 0.057 \\
\hline Total cryotherapy time per vein, $s$ & $244.0 \pm 45.5$ & $375.0 \pm 45.0$ & $<0.001$ \\
\hline 2 lesions per vein, $n$ (\%) & $70(87.50 \%)$ & $71(84.52 \%)$ & 0.583 \\
\hline Late/ATP reconnection,n (\%) & $1(1.25 \%)$ & $3(3.57 \%)$ & 0.648 \\
\hline \multicolumn{4}{|l|}{ Vein(RSPV) } \\
\hline PV potentials, n (\%) & $66(82.50 \%)$ & $68(80.95 \%)$ & 0.798 \\
\hline TT-PVI, s & $39.7 \pm 18.9$ & $40.2 \pm 16.9$ & 0.862 \\
\hline Nadir temperature, ${ }^{\circ} \mathrm{C}$ & $-52.8 \pm 4.4$ & $-51.6 \pm 3.8$ & 0.061 \\
\hline Total cryotherapy time per vein, $s$ & $236.8 \pm 41.9$ & $366.7 \pm 21.2$ & $<0.001$ \\
\hline 2 lesions per vein, $n$ (\%) & $70(87.50 \%)$ & 75(89.29\%) & 0.721 \\
\hline Late/ATP reconnection,n (\%) & 0 & $2(2.38 \%)$ & 0.497 \\
\hline \multicolumn{4}{|l|}{ Vein(RIPV) } \\
\hline PV potentials, n (\%) & $57(71.25 \%)$ & $60(71.43 \%)$ & 0.980 \\
\hline TT-PVI, s & $45.4 \pm 23.6$ & $46.8 \pm 19.2$ & 0.722 \\
\hline Nadir temperature, ${ }^{\circ} \mathrm{C}$ & $-49.7 \pm 4.8$ & $-46.8 \pm 5.9$ & 0.001 \\
\hline Total cryotherapy time per vein, $s$ & $248.1 \pm 40.3$ & $392.0 \pm 60.8$ & $<0.001$ \\
\hline 2 lesions per vein, $n$ (\%) & $62(77.50 \%)$ & $60(71.43 \%)$ & 0.373 \\
\hline Late/ATP reconnection,n (\%) & $1(1.25 \%)$ & $3(3.57 \%)$ & 0.648 \\
\hline
\end{tabular}


Values are given as mean \pm SD or $\mathrm{n}(\%)$, unless otherwise indicated. LIPV=left inferior pulmonary vein; LSPV=left superior pulmonary vein; PV=pulmonary vein; RIPV=right inferior pulmonary vein; RSPV=right superior pulmonary vein; TT-PVI=time to pulmonary vein isolation.

Table 4 Major complications

\begin{tabular}{llll}
\hline Adverse events & Cryo-AF $_{\text {Dosing }}(\mathrm{n}=80)$ & $\mathrm{Cryo}_{-\mathrm{AF}_{\text {Conventional }}(\mathrm{n}=84)}$ & $P$ \\
\hline All, n (\%) & $4(5 \%)$ & $6(7.14 \%)$ & 0.566 \\
Groin vascular complications, $\mathrm{n}(\%)$ & $3(3.75 \%)$ & $4(4.76 \%)$ & 0.749 \\
Pericardial effusion, n (\%) & 0 & $1(1.19 \%)$ & 0.328 \\
Phrenic nerve palsy, n (\%) & $1(1.25 \%)$ & $1(1.19 \%)$ & 0.972 \\
\hline
\end{tabular}

Values are given as n (\%)

Table 5 Comparison of freedom from recurrent atrial arrhythmias between the two groups

\begin{tabular}{llll}
\hline & $\mathrm{Cryo}_{-} \mathrm{AF}_{\text {Dosing }}(\mathrm{n}=80)$ & $\mathrm{Cryo}_{-} \mathrm{AF}_{\text {Conventional }}(\mathrm{n}=84)$ & $P$ \\
\hline At 3 months & $73(91.25 \%)$ & $75(89.29 \%)$ & 0.672 \\
At 6 months & $68(85.00 \%)$ & $70(83.33 \%)$ & 0.770 \\
At 9 months & $65(81.25 \%)$ & $68(80.95 \%)$ & 0.961 \\
At 12 months & $63(78.75 \%)$ & $66(78.57 \%)$ & 0.978 \\
\hline
\end{tabular}

Values are given as $\mathrm{n}(\%) . \mathrm{Cryo}-\mathrm{AF}=$ cryoablation of atrial fibrillation

Figures 


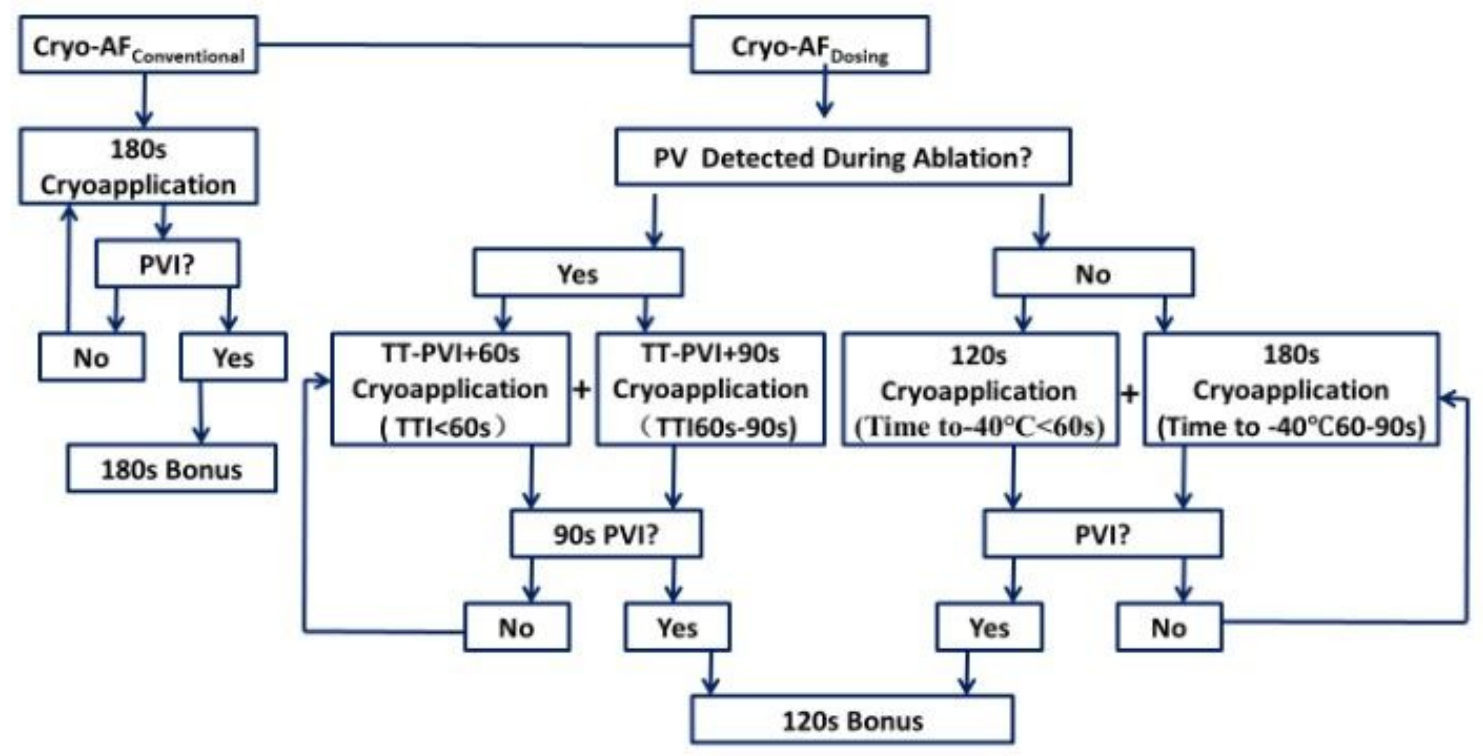

\section{Figure 1}

Protocol flowchart depicting cryoenergy dosing performed according to the TT-PVI or balloon temperature (Cryo-AFDosing group) or conducted with a conventional ablation protocol (Cryo-AFConventional group). In the Cryo-AFConventional group, cryoablation of 180 seconds was applieduntil bidirectional block of the vein was demonstrated. Following successful PVI, an additional 180-second freeze cycle was applied. In the Cryo-AFDosing group, the freeze duration was modified depending on the observed TT-PVI or balloon temperature. If a spontaneous TT-PVI was detectable, cryoenergy applications lasted for the TT-PVI plus $60-90$ seconds. If the TT-PVI was $<60$ seconds, a TT-PVI plus 60 seconds freeze cycle was applied. If the TT-PVI was between 60 and 90 seconds, a TT-PVI plus 90 seconds freeze cycle was applied.

Additionally,if the TT-PVI was unavailable, according to the time from the beginning of ablation to the balloon temperature achievement of $-40^{\circ} \mathrm{C}$, cryoenergy of 120-180 seconds was applied until the vein was blocked. In other words, if the temperature reached $-40^{\circ} \mathrm{C}$ within 60 seconds, a 120-second freeze cycle was applied, and if the temperature reached $-40^{\circ} \mathrm{C}$ within $60-90$ seconds, a 180 -second freeze cycle was applied. In both cases, a 120-second bonus application was immediately administered. Cryo$\mathrm{AF}=$ cryoablation of atrial fibrillation; $\mathrm{PV}=$ pulmonary vein; $\mathrm{TT}-\mathrm{PVI}=$ time-to-pulmonary vein isolation. Time to $-40^{\circ} \mathrm{C}$, defined as the balloon freezing time from the beginning of the application to the moment of achievement of $-40^{\circ} \mathrm{C}$. 


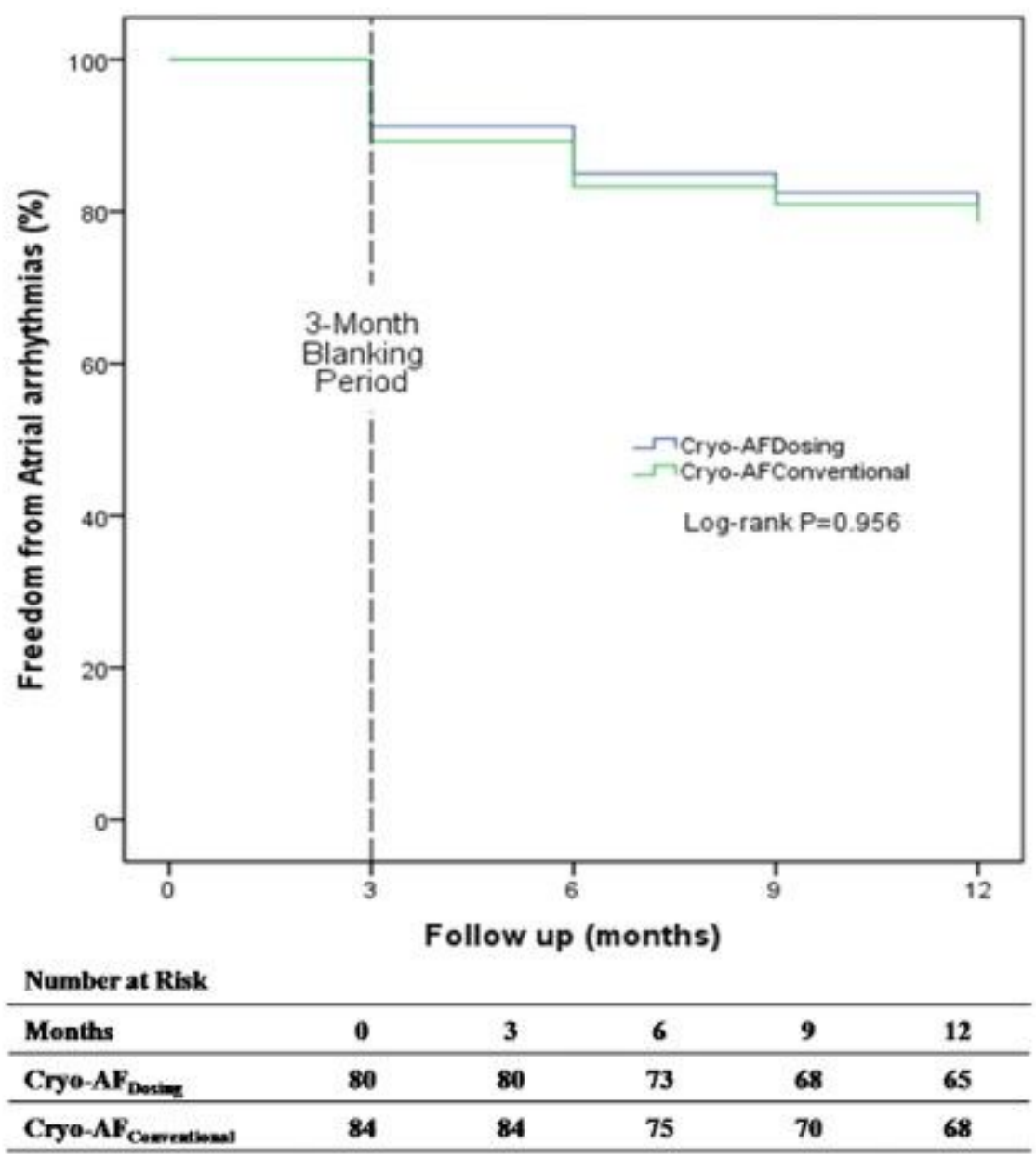

Figure 2

Kaplan-Meier curves indicating freedom from atrial arrhythmia recurrence during follow-up after cryoballoon pulmonary vein isolation with a Cryo-AFDosing group or a Cryo-AFConventional group. 